\title{
Relapse in a borderline-tuberculoid case of leprosy 5 years after the release from rifampicin monotherapy
}

V. P. SHETTY \& N. H. ANTIA

The Foundation for Medical Research, 84-A, R. G. Thadani Marg, Worli, Bombay 400 018, India

Accepted for publication 5 February 1997

\section{Introduction}

Paucibacillary cases of leprosy are now routinely being treated with two drugs, namely rifampicin (RFP) and diamino diphenyl sulphone (DDS). We report here a paucibacillary case of borderline-tuberculoid (BT) leprosy, who had taken a one-year course of daily RFP monotherapy, and relapsed as BT five years after the release from treatment. Mycobacterium leprae derived from this case was sensitive to both $0.01 \mathrm{~g} \%$ DDS and $0.03 \mathrm{~g} \%$ RFP.

\section{Case report}

A 67-year-old unmarried female was referred to us complaining of the reappearance and increase in size of an old patch on the dorsum of her left leg. The history goes as follows.

In the year 1981 she noticed a coin-sized patch on the dorsum of the left leg. In 1983 she was diagnosed as having leprosy, was smear negative and was put on DDS and RFP. She developed a severe allergy to dapsone and this was withdrawn within a week, and she was maintained on RFP alone. The patient continued with daily $600 \mathrm{mg}$ RFP for one full year. She was fair skinned and was unwilling to take clofazimine as advised by the referring doctor. The patch subsided completely and the patient remained symptom free till 1989. In that year the patch reappeared, remained static till around 1992, and slowly increased in size thereafter. Upto April 1995 the patient only used a local antihistamine ointment.

On examination the old patch had increased three-fold in size, and was well defined, erythematous and anaesthetic. The left sural nerve was palpably thick and slightly tender. There was no other apparent patch or nerve involvement. Lepromin given at this stage was reading $4.9 \mathrm{~mm}$ at three weeks.

\section{Investigations}

The skin patch and the sural nerve were biopsied under local anaesthesia. Part of both skin and nerve were fixed in Formal Zenker and processed for histology. Other portions of the 
biopsies were homogenized for bacterial count and injected into the footpads of normal Swiss white (SW) mice. Tests for sensitivity to $0.01 \mathrm{~g} \%$ DDS and $0.03 \mathrm{~g} \%$ RFP were set up along with a control group of mice using the standard protocol. ${ }^{1}$

Part of the nerve biopsy was also fixed in glutaraldehyde and processed for electron microscopy.

\section{Results}

\section{HISTOPATHOLOGY}

Skin: The skin patch biopsy sections stained with Trichrome modified Fite Faracco (TRIFF) showed flattened retepegs and normal keratinization. In the superficial dermis and around the adnexa a moderate degree of loosely arranged oedematous infiltrate consisting of epitheloid cells and scattered lymphocytes was seen. One deep dermal nerve was replaced by epitheloid cells. No acid-fast bacilli were detected in the section.

Nerve: In the TRIFF-stained section, there were multiple fascicles and two of them were grossly infiltrated. The infiltrate was more organized, in which epitheloid cells were seen surrounded by a moderate number of lymphocytes in the endoneurium.

Araldite-embedded $1-\mu$ thick section of nerve (TS) stained with toluidine blue showed six fascicles, of which three were partially involved. Active epitheloid type macrophages surrounded by lymphocytes, a few plasma cells and mast cells were seen in the endoneurium. The infiltrated area showed severe fibre dropout (see Figure 1).

Ultrathin sections stained with uranyl acetate and lead citrate were scanned using a transmission electron microscope. The presence of a fair number of bacteria, either singly or in small clusters were seen predominantly in the nonmyelinated fibre Schwann cells (Figure 2) in all the three involved fascicles. Both skin and nerve pathology were thus consistent with a borderline-tuberculoid type of lesion.

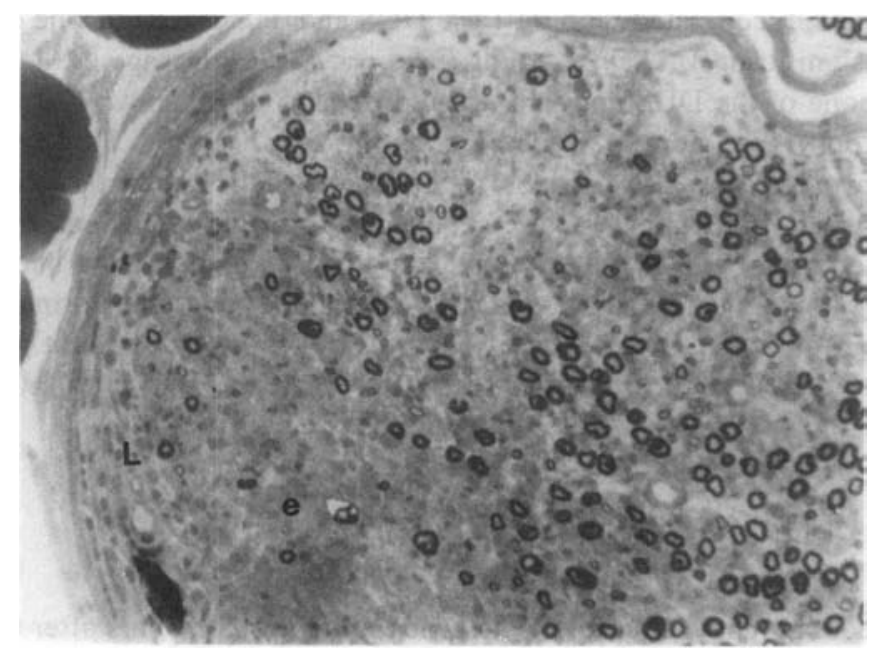

Figure 1. Part of one of the involved fascicles of the sural nerve in T.S. showing drop out of myelinated fibres in the infiltrated area. Note the presence of epitheloid cells (e) and lymphocytes (L) in the periphery of the lesion. Araldite embedded one-micron-thick section stained with toluidine blue $\times 120$. 


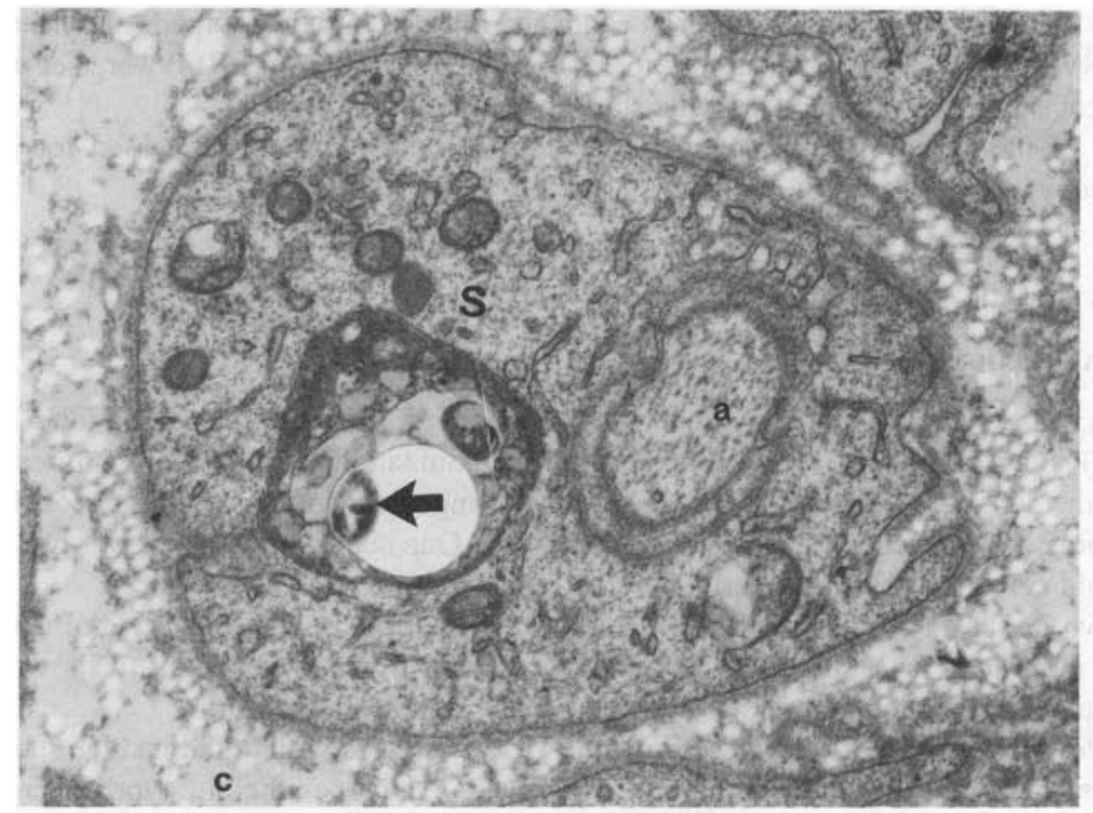

Figure 2. Part of the nerve shown in Figure 1 under the electron microscope. Note the presence of bacillus $(\rightarrow)$ within a phagosome in the Schwann-cell cytoplasm(s). a, axon; c, collagen; $\times, 16,000$.

\section{Bacterial load in skin and nerve, viability and sensitivity to drugs}

Skin biopsy homogenate showed no detectable M. leprae count in over 200 fields on a spot slide. Multiple harvests were carried out at 12 months after the footpad inoculation from both control (No Rx) and mice treated with 0.01 g\% DDS and 0.03 g\% RFP. The control group of mice showed a significant $M$. leprae count (take $=5 / 7$ and average $M$. leprae count was $6 \times 10^{5}$ ), while none of the DDS and RFP treated mice ( 5 each) showed any count.

The nerve biopsy homogenate yielded a count of ' $1 \times 10^{6} \mathrm{~g} / \mathrm{wt}$ '. However, it was noted that the bacteria were very weakly acid fast (stained with Ziehl Neelsen's cold method). Both skin and nerve biopsy homogenates were streaked onto Lowenstein Jensen media and soft agar plate, showed no growth in either, thus excluding any contaminant bacteria.

Nerve biopsy homogenate that was injected into the footpads of normal SW mice also showed $M$. leprae multiplication in all the six control mice (average count was $2 \times 10^{6}$ ) and none in the drug treated mice ( 5 each), proving to be sensitive to both $0.01 \mathrm{~g} \%$ DDS and $0.03 \mathrm{~g} \%$ RFP.

\section{Discussion}

This is a paucibacillary case of leprosy who probably relapsed 5 years after the release from RFP monotherapy and remained untreated for the following 5 years. It is noteworthy that the reactivation of the old skin lesion occurred 5 years after release from treatment, remained static for another 3 years and then increased in size. Histopathology of both skin patch and 
involved sural nerve, obtained 7 years after the first sign of reactivation, confirmed borderline-tuberculoid leprosy.

The first question that arises is, could it be a case of reinfection? Circumstances of relapse in this case, that is the reactivation followed by increase in size of the old lesion and the patients very sedentary life style for the past 10 years are strongly in favour of relapse due to persisters and as such this may not be an isolated incidence.

Persistence of viable bacteria in this case could be attributed to the inappropriate treatment regimen, i.e. use of RFP monotherapy. This indicates that one year of $600 \mathrm{mg}$ daily RFP failed to eliminate a bacterial load of approximately less than $10^{6}$. Since M. leprae when tested were sensitive to RFP, it appears that the RFP failed to act on nondividing bacilli and it may be that the addition of a bacteriostatic drug such as DDS may also prove counterproductive.

It was interesting to note that even though the skin patch did not show any bacilli either in the histology or in the homogenate, a significant yield was obtained in the footpad of normal SW mice at the 12th month, albeit delayed and lower than the one obtained with the nerve homogenate, thus demonstrating that the skin lesion also harboured viable bacteria, either in a very small number or they were nonacid-fast. ${ }^{2}$ The second explanation derives further support from the observation made on the nerve homogenate of this patient, where the bacilli were found to be very weakly acid fast, to the extent that we ourselves initially thought that they might be contaminant bacteria. However, lack of growth in the LJ slope and on soft agar plating, confirmation using electron microscopy of the presence of bacilli in the Schwann-cell cytoplasm, which remains a unique characteristic of $M$. leprae, ${ }^{3}$ and the subsequent growth in the footpad of the mouse confirm all the characteristics of $M$. leprae and indicate that these were viable in both the skin and nerve lesions of this patient.

Another point that needs to be reiterated is that the clinical findings or even the histopathology of the skin would have led to a wrong conclusion of late reversal reaction. ${ }^{4,5}$ Even though the time course favours relapse, in the absence of any demonstrable acid-fast organism very often such cases are considered for steroid therapy without the coverage of antileprosy treatment. ${ }^{6}$ The mouse footpad results obtained in the present study is strongly in support of the view held by a few that when there is any evidence of deteriorating neural symptoms, it is very likely a case of relapse. ${ }^{7}$ In the present case the sural nerve probably acted as a bacterial repository.

Treatment and progress: Following the biopsy the patient was put on ofloxacin- $450 \mathrm{mg}$ (BD) and rifampicin $450 \mathrm{mg}$ (OD) for one month followed by RFP (OD) for 6 months. The response remains good up till now.

\section{Acknowledgments}

We thank Dr Swaran Arora of Tata Department of Plastic Surgery, J. J. Group of Hospitals, Bombay, for referring the patient, Dr Satish Arolkar for doing the biopsy and Ms Anju Dighe for technical assistance.

\section{References}

1 World Health Organization, 1987, Laboratory techniques for leprosy.

2 Barros U, Shetty VP, Antia NH. Demonstration of $M$. leprae antigen in nerves of tuberculoid leprosy. Acta Neuropathol 1987; 73: 387-392. 
3 Antia NH. Leprosy-A disease of the Schwann cell. Ind J Lepr 1982; 54: 593-604.

${ }^{4}$ Panikar V, Jesudasan K, Vijayakumaran P, Christian M. Relapse or late reversal reaction. Int J Lepr 1989; 57: 526-528.

5 Ramachandran R, Seshadri PS. Relapse or reversal reaction: The case for a therapeutic trial of steroids. Lepr Rev 1988; 59: 271-272.

${ }_{7}^{6}$ Pfaltzgraff RE. Management of reactions in leprosy. Int J Lepr 1983; 57: 103-109.

7 Kesava Reddy P, Cherian A. Relapse in leprosy after multi drug therapy and its differential diagnosis with reversal reaction. Ind J Lepr 1991; 63(1): 61-69. 